\title{
A REMARK ON THE DENSITY CHARACTER OF FUNCTION SPACES
}

\author{
GIOVANNI VIDOSSICH
}

Concerning the density character of function spaces, the most general theorem we know is the unique theorem in [4] - whose brilliant proof carries over verbatim to arbitrary infinite cardinalsand its generalization in $[5,(\mathrm{~J})$ and $(\mathrm{D})]$. The present note investigates the case of the range space separable without countable base as in Michael's theorem [4]. A positive result is given for the range a convex subset of a locally convex space, or an injective uniform space.

The author wishes to thank the referee for helpful suggestions.

Notations. We shall denote by

$C(X, Y)$ the set of all continuous maps $X \rightarrow Y$.

$C_{\mathrm{c}}(X, Y)$ the set $C(X, Y)$ with the compact-open topology.

$C_{\mathrm{p}}(X, Y)$ the set $C(X, Y)$ with the product topology.

$w(X)$ the weight of $X=$ least cardinal of a basis for the topology of $X$.

$\operatorname{dc}(X)$ the density character of $X=$ least cardinal of a dense subset of $X$.

THEOREM. Let $X$ be a topological space and $Y$ a convex subset of a locally convex space $Z$. If $Y$ is equipped with the induced topology, then

$$
\operatorname{dc}\left(C_{\mathrm{c}}(X, Y)\right) \leqq w(X) \cdot \operatorname{dc}(Y)
$$

provided that $\mathrm{w}(X)$ or $\mathrm{dc}(Y)$ is infinite.

Proof. Because a translation is a homeomorphism preserving convexity, we may assume as we do-that $0 \in Y$. Let $I^{n}$ be the product of the unit interval $n$ times $\left(n \in Z^{+}\right)$, and let $J_{n}$ be the subset of $I^{n}$ defined by the condition: $x \in J_{n}$ iff the sum of the coordinates of $x$ is less than or equal to 1 . Because $Y$ is convex and $0 \in Y$, there is a natural map $\Phi_{n}: C_{\mathrm{c}}\left(X, J_{n}\right) \times Y^{n} \rightarrow C_{\mathrm{c}}(X, Y)$ defined by

$$
\Phi_{n}(f, y)=\left(x \mapsto \sum_{i=1}^{n} f_{i}(x) y_{i}\right)
$$

where $f=\left(f_{1}, \cdots, f_{n}\right) \in C\left(X, J_{n}\right)$ and $y=\left(y_{1}, \cdots, y_{n}\right) \in Y^{n}$. Clearly $\Phi_{n}$ is continuous. From the fact that compact $T_{3}$-spaces (as all compact subsets of $X$ are under the weak topology determined by an $f \in C(X, Y)$ ) admit partitions of unity and from the fact that $Y$ is $\mathrm{AE}$ (metric)-hence $\mathrm{AE}$ (pseudometric)—by Dugundji extension theo-

Received by the editors August 29, 1968 and, in revised form, January 13, 1969. 
rem [2, ix.6.1], it is easily seen that $\bigcup_{n=1}^{\infty} \Phi_{n}\left(C_{\mathrm{c}}\left(X, J_{n}\right) \times Y^{n}\right)$ is dense in $C_{\mathbf{c}}(X, Y)$. By the same proof of the unique theorem in [4] we may establish the following theorem:

If $\aleph$ is an infinite cardinal and $X, Y$ are arbitrary topological spaces with $w(X), w(Y) \leqq \aleph$, then hereditarily $\mathrm{dc}\left(C_{\mathrm{c}}(X, Y)\right) \leqq \aleph$.

This theorem implies that $\operatorname{dc}\left(C_{\mathrm{c}}\left(X, J_{n}\right)\right) \leqq w(X) \cdot \boldsymbol{\aleph}_{0}$ because $J_{n}$ is separable metric. Moreover, $\operatorname{dc}\left(Y^{n}\right) \leqq \operatorname{dc}(Y) \cdot \boldsymbol{\aleph}_{0}$. Therefore, by the continuity of $\Phi_{n}$,

$$
\operatorname{dc}\left(\Phi_{n}\left(C_{\mathrm{c}}\left(X, J_{n}\right) \times Y^{n}\right)\right) \leqq w(X) \cdot \operatorname{dc}(Y) \cdot \boldsymbol{\aleph}_{0}^{2}=w(X) \cdot \operatorname{dc}(Y) .
$$

Hence the result. Q.E.D.

The above result is poor in the case of $X$ discrete: If $\operatorname{Card}(X)=2^{\aleph_{0}}$, then $C_{\mathrm{c}}(X, R)$ is separable by $[2$, viii.7.2.(3)], although the above theorem only gives a dense subset of cardinality $\leqq 2 \aleph_{0}$. But the above theorem is best possible in case $X$ is compact: If $X$ is the product of the unit interval $2 \aleph_{0}$ times, then $X$ is compact, separable (by [2, viii.7.2.(3) ]) and nonmetrizable; therefore $w(X)=2^{\aleph_{0}}$, and $C_{\mathrm{c}}(X, R)$ cannot be separable, because otherwise $X$ would be metrizable by $[1, \S 3$, Theorem 1$]$.

The property of the above theorem is not hereditary: Let $K$ be the Cantor space. Because $K$ is uncountable, $C_{\mathrm{p}}\left(K, C_{\mathrm{p}}(K,[0,2])\right)$ is not hereditarily separable as the proof of $[5$, Lemma 10.6] indicates. And $\left.C_{\mathrm{p}}(K,[0,2])\right)$ is a separable convex subset of a locally convex space - the product $R^{K}$.

A natural question: Is the above theorem true for general $Y$ ? A positive answer can be derived as a corollary of the above theorem via a uniform embedding into a product of Banach spaces when $Y$ is an injective uniform space for some uniformity (according to [3, p. 39], a uniform space $Y$ is called injective iff, whenever $A$ is a uniform subspace of $X$, every uniformly continuous map $A \rightarrow Y$ can be extended to a uniformly continuous map $X \rightarrow Y$ ).

\section{REFERENCES} 1961.

1. N. Bourbaki, Topologie générale, Chapter 10, 2nd rev. ed., Hermann, Paris,

2. J. Dugundji, Topology, Allyn and Bacon, Boston, Mass., 1966.

3. J.IR. Isbell, Uniform spaces, Math. Surveys, No. 12, Amer. Math. Soc., Providence, R. I., 1964. 921.

4. E. Michael, On_a theorem of Rudin and Klee, Proc. Amer. Math. Soc. 12 (1961),

5. - $\aleph_{0}$-spaces, J. Math. Mech. 15 (1966), 983-1002.

Libero Pensatore 